\title{
Origin discrimination and quality evaluation of Gastrodiae rhizoma (Orchidaceae) by high-performance liquid chromatographic fingerprint
}

\author{
Rui-xue Zhong1, Guang-fu Feng', Yu-jie Liü, Lian Zhong ${ }^{2}$, Chun-jie $\mathbf{W u}^{2}$ and \\ Jun Wan ${ }^{3 *}$ \\ ${ }^{1}$ Sichuan Provincial Orthopedic Hospital, Chengdu, 610000, ${ }^{2}$ School of Pharmacy, Chengdu University of Traditional Chinese \\ Medicine, Chengdu, 610075, ${ }^{3}$ Life Science \& Engineering College of South-west Jiao-tong University, Chengdu, 610031, PR \\ China
}

*For correspondence: Email: wanjuntcm@163.com; Tel/Fax: +86-13980561562

\begin{abstract}
Purpose: To develop a high-performance liquid chromatography (HPLC) fingerprint method for the quality control and origin discrimination of Gastrodiae rhizoma.

Methods: Twelve batches of G. rhizoma collected from Sichuan, Guizhou and Shanxi provinces in china were used to establish the fingerprint. The chromatographic peak (gastrodin) was taken as the reference peak, and all sample separation was performed on a Agilent C18 (250 mm×4.6 mmx5 $\mu \mathrm{m}$ ) column with a column temperature of $25^{\circ} \mathrm{C}$. The mobile phase was acetonitrile/0.8 \% phosphate water solution (in a gradient elution mode) and the flow rate of $1 \mathrm{~mL} / \mathrm{min}$. The detection wavelength was 270 $n m$. The method was validated as per the guidelines of Chinese Pharmacopoeia.

Results: The chromatograms of the samples showed 11 common peaks, of which no. 4 was identified as that of Gastrodin. Data for the samples were analyzed statistically using similarity analysis and hierarchical cluster analysis (HCA). The similarity index between reference chromatogram and samples' chromatograms were all $>0.80$. The similarity index of G. rhizoma from Guizhou, Shanxi and Sichuan is evident as follows: $0.854-0.885,0.915-0.930$ and $0.820-0.848$, respectively. The samples could be divided into three clusters at a rescaled distance of 7.5: S1 - S4 as cluster 1; S5 - S8 cluster 2, and others grouped into cluster 3.

Conclusion: The findings indicate that HPLC fingerprinting technology is appropriate for quality control and origin discrimination of G. rhizoma.
\end{abstract}

Keywords: Gastrodiae rhizoma, Origin discrimination, Quality control; HPLC fingerprint

Tropical Journal of Pharmaceutical Research is indexed by Science Citation Index (SciSearch), Scopus, International Pharmaceutical Abstract, Chemical Abstracts, Embase, Index Copernicus, EBSCO, African Index Medicus, JournalSeek, Journal Citation Reports/Science Edition, Directory of Open Access Journals (DOAJ), African Journal Online, Bioline International, Open-J-Gate and Pharmacy Abstracts

\section{INTRODUCTION}

Gastrodiae rhizoma (Tianma in Chinese), the dry tuber of Gastrodia elata Blume, is a commonused and extraordinary TCM listed in the Chinese Pharmacopoeia 2015 edition [1]. In Han Dynasty, it's typically recorded as a high quality drug in Shen-nung-pen-tsao-ching to treat headache, anticonvulsant, paralysis, epilepsy and tetanus $[2,3]$. The $G$. rhizoma was mainly planted in Sichuan, Guizhou and Shanxi, etc. in China.

As a precious and commonly used medicinal material, the quality control of G. rhizoma is greatly necessary. Though some quality control methods were established, previous investigations on quality control of G. rhizoma 
mostly focused on determination of single or several major compounds $[4,5]$.

In addition, different origin area and growing conditions may cause significant differences in chemical patterns and quality of $G$. rhizoma $[6,7]$. However, it is very difficult to divide G. rhizoma from different areas in appearance. Therefore, it is important to develop a method for better quality control and the origin discrimination of $G$. rhizoma.

High-performance liquid chromatography (HPLC) fingerprinting technology is proved to be a useful tool for qualitative and quantitative analysis in TCM, especially some unknown components in herbal drugs [8-10]. To obtain the meaningful correlations from HPLC fingerprint, statistical analysis based on the samples' data are usually required, and similarity analysis and hierarchical cluster analysis (HCA) are the most popular statistical methods $[11,12]$.

In the present study, HPLC was used to establish the chromatographic fingerprint for quality control and origin discrimination of $G$. rhizoma by combining the chemometric methods including similarity evaluation and HCA.

\section{EXPERIMENTAL}

\section{Chemicals and reagents}

Gastrodin (Batch No.110807-200205) was purchased from National Institutes for Food and Drug Control (Beijing, China). Chromatographicgrade acetonitrile and methanol were all purchased from Fisher Chemicals (Fisher lawn, New Jersey, USA). The water used in the experiment was made by the Millipore purification system (Millipore, MA, USA). All the other reagents used in the experiment were of analytical grade.

Table 1: The samples of $G$. rhizoma used in this study

\section{Sample preparation}

G. rhizoma were collected from Sichuan, Guizhou, Shanxi, and identified by Professor Liang-ke Song in South-west Jiao-tong University. Then, the samples were pulverized into powder and sifted through the standard 50mesh sieve $(355 \pm 13 \mu \mathrm{m})$. The information on the samples are listed in Table 1.

The 12 pulverized samples of $\mathrm{G}$. rhizoma were accurately weighed (approximately $4.0 \mathrm{~g}$ ) and the samples were extracted by reflux for $1 \mathrm{~h}$ in $50 \mathrm{~mL}$ of methanol. Then, the extracted solutions were filtered through a $0.22 \mu \mathrm{m}$ filtration membrane, and the injection volume was $10 \mu \mathrm{L}$. Gastrodin standard was dissolved with methanol as the standard solution.

\section{HPLC chromatographic conditions}

HPLC was performed using an Agilent 1200 high performance liquid chromatography system (Agilent Technologies, CA, USA). The separation of samples was performed on an Agilent C18 (250 mm×4.6 mm, $5 \mu \mathrm{m}$, Agilent Company) column. The mobile phase consisted of $(A)$ phosphate acid/water $(0.4: 100, v / v)$ and (B) acetonitrile. The most suitable linear gradient elution program was shown in Table 2. The flow rate was $1.0 \mathrm{~mL} / \mathrm{min}$, and the temperature of the chromatographic column was maintained at 25 ${ }^{\circ} \mathrm{C}$. The wavelength selected for our experiment was $270 \mathrm{~nm}$.

\section{Data analysis}

Similarity analysis was performed by using the Similarity Evaluation System for Chromatographic Fingerprint of Traditional Chinese Medicine (Version 2004A), which is recommended by Chinese Pharmacopoeia Commission. The hierarchical cluster analysis of samples was performed by SPSS software (SPSS for Windows 17.0, SPSS Inc., USA).

\begin{tabular}{cllc}
\hline Sample no. & Herbal species & Collection area & Collection time \\
\hline S1 & Gastrodia elata Blume & Da Fang, Guizhou Province & $23-10-2012$ \\
S2 & Gastrodia elata Blume & Da Fang, Guizhou Province & $23-10-2012$ \\
S3 & Gastrodia elata Blume & Da Fang, Guizhou Province & $23-10-2012$ \\
S4 & Gastrodia elata Blume & Da Fang, Guizhou Province & $23-10-2012$ \\
S5 & Gastrodia elata Blume & MianYang, Sichuan Province & $15-11-2012$ \\
S6 & Gastrodia elata Blume & MianYang, Sichuan Province & $15-11-2012$ \\
S7 & Gastrodia elata Blume & MianYang, Sichuan Province & $15-11-2012$ \\
S8 & Gastrodia elata Blume & MianYang, Sichuan Province & $15-11-2012$ \\
S9 & Gastrodia elata Blume & Han Zhong, Shanxi Province & $18-12-2012$ \\
S10 & Gastrodia elata Blume & Han Zhong, Shanxi Province & $18-12-2012$ \\
S11 & Gastrodia elata Blume & Han Zhong, Shanxi Province & $18-12-2012$ \\
S12 & Gastrodia elata Blume & Han Zhong, Shanxi Province & $18-12-2012$ \\
\hline
\end{tabular}


Table 2: Linear gradient elution program for the mobile phase

\begin{tabular}{lcc}
\hline $\mathbf{T}$ (min) & A (\%) & B (\%) \\
\hline $0-10$ & $98-98$ & $2-2$ \\
$10-20$ & $98-96$ & $2-4$ \\
$20-25$ & $96-96$ & $4-4$ \\
$25-35$ & $96-89$ & $4-11$ \\
$35-50$ & $89-89$ & $11-11$ \\
$50-60$ & $89-82$ & $11-18$ \\
$60-78$ & $82-82$ & $18-18$ \\
\hline
\end{tabular}

\section{RESULTS}

\section{Optimized chromatographic conditions}

In this study, the mobile phase was investigated on the chromatographic separation of the samples. The different mobile phase compositions were as follows: acetonitrilephosphoric acid/water $(0.1: 100, \mathrm{v} / \mathrm{v})$, acetonitrilephosphoric acid/water $(0.4: 100, \mathrm{v} / \mathrm{v})$, methanolphosphoric acid/water $(0.1: 100, \mathrm{v} / \mathrm{v})$, methanolphosphoric acid/water $(0.4: 100, \mathrm{v} / \mathrm{v})$. As a result, the acetonitrile-phosphoric acid/water $(0.4: 100$, $\mathrm{v} / \mathrm{v}$ ) was selected as an appropriate mobile phase with best resolution and shortest analysis time. Selection of an appropriate detection wavelength plays an important role to ensure precise detection of some essential constituents and to achieve more peaks. Diode Array Detector was used in the present study, and full scan runs were made initially to select the optimum wavelength that provided the best results in chromatographic fingerprinting analysis. The chromatogram obtained at $270 \mathrm{~nm}$ showed the steadiest baseline and the most abundant components information comparing with the other wavelengths. As a result, $270 \mathrm{~nm}$ was chosen as the monitoring wavelength.

\section{Optimized of extracting conditions}

\section{Extraction solvent}

The samples were subjected to reflux extraction with ethanol, water and methanol, respectively. The results showed that methanol extract had more number of peaks than other solvent. Finally, methanol was finally chosen as the optimal extracting solvent.

\section{Extraction method}

With methanol as the extraction solvent, the samples were extracted by reflux and by ultrasonic in comparison. According to the results of two methods, the more peaks were obtained from reflux extraction. Consequently, the reflux extraction was chosen as extracting method for the samples.

\section{Validation}

The method was validated as per the guideline of Chinese Pharmacopoeia [13].The injection precision was determined through replicating the same sample solution and continually injected six times per day. The sample stability was determined by taking one sample solution kept at room temperature for $0,4,8,12,18$ and $24 \mathrm{~h}$. The repeatability of this test was estimated by analyzing six separate extract solutions of one sample. In terms of injection precision, the relative standard deviation (RSD) of RRT and RPA were below 0.95 and $2.83 \%$, respectively; In terms of sample stability, the RSD values were found not to exceed 1.45 and $2.88 \%$, respectively; In terms of repeatability, the RSD values were less than $0.66 \%$ and $2.86 \%$, respectively. Therefore, all the results indicate that the established method is stable and under control.

\section{HPLC fingerprint}

The chromatograms of the twelves samples were recorded in 78 minutes, which were shown in Figure 1. According to the results of HPLC fingerprint, 11 common peaks were found. Among the characteristic peaks, peak No. 4 at retention time of 17.56 minutes was identified as Gastrodin compared with the Gastrodin standard, and it was designated as the reference peak. Because it appears with a stable time and a large area in the chromatogram, the RSD value is below $3.0 \%$. Also its comparative standing time of common peaks approximately fits the requirements.

Gastrodin is one of the main active components in G. rhizoma, featuring with broad pharmacological activity, sedative, anticonvulsant actions [14]. It is often prescribed to treat vertigo, paralysis, hemiplegia and rheumatism diseases. Thus, as an object of reference, Gastrodin can be used in the identification and quality evaluation of G. rhizoma.

\section{Relative peak area and similarity of the samples}

The chromatograms of twelves sample were recorded at $270 \mathrm{~nm}$ and the chromatographic peaks in twelves samples with the same relative retention time were defined as the characteristic peaks. Eleven peaks were identified as the 


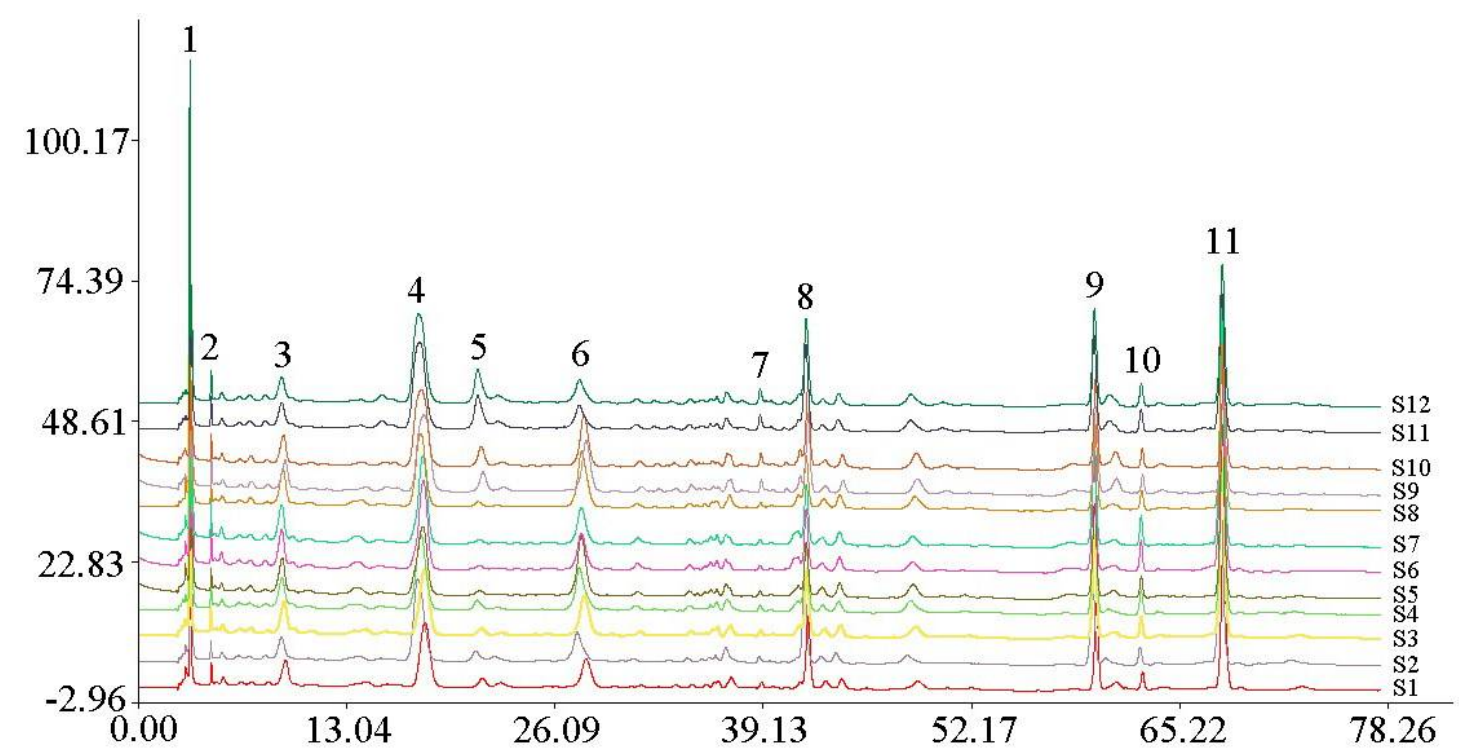

Fig 1: Chromatogram of the fingerprints of the twelves samples of G. rhizoma

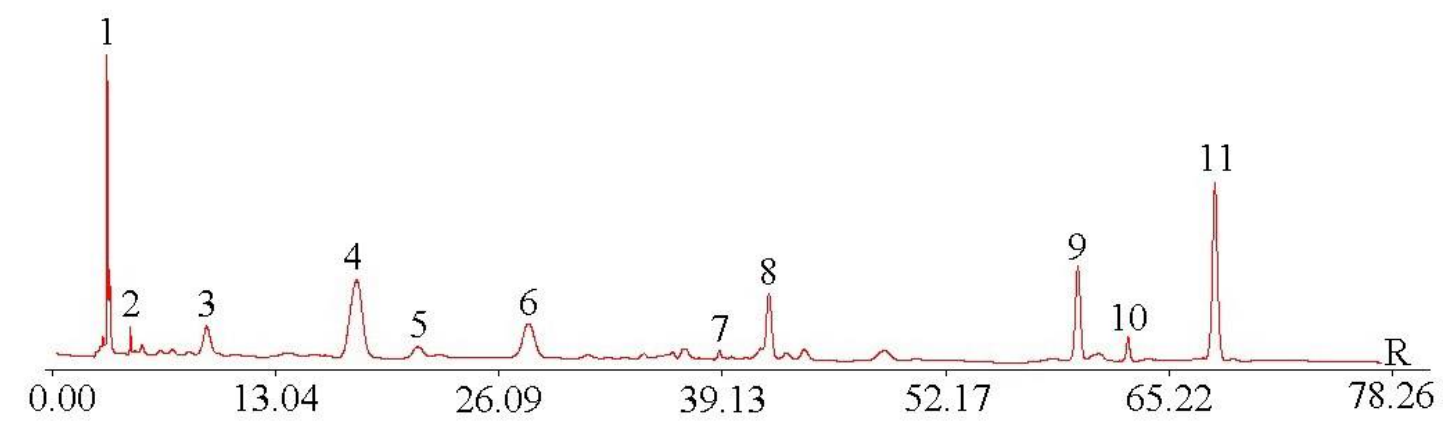

Figure 2: Chromatogram of the fingerprints of the reference chromatogram

characteristic peaks. Gastrodin was designated as the reference peak for the calculation of RPA. Moreover, the relative retention time (RRT) and relative retention area (RPA) of these 11 peaks in twelve samples were shown in Table 3 . The data of the chromatographic fingerprints were analyzed by the Similarity Evaluation System for Chromatographic Fingerprint of Traditional Chinese Medicine (Version 2004 A) software (Chinese Pharmacopoeia Commission, Beijing, China). The reference chromatogram was generated by the median method and the similarity indexes of the twelves samples are listed in Table 4.

\section{Similarity index}

As shown in Figure 3, compared with the reference chromatogram created via the median method, the similarity of the twelves samples gathered from three different provinces (Sichuan, Guizhou and Shanxi) in China were all above

Table 3: Calculated relative peak area of the communion peak

\begin{tabular}{|c|c|c|c|c|c|c|c|c|c|c|c|c|}
\hline Code & $\overline{\text { S1 }}$ & $\overline{S 2}$ & $\overline{S 3}$ & $\overline{S 4}$ & S5 & 56 & S7 & S8 & S9 & S10 & S11 & $\mathrm{S12}$ \\
\hline 1 & 0.496 & 0.456 & 0.514 & 0.548 & 0.329 & 0.337 & 0.313 & 0.369 & 0.255 & 0.252 & 0.403 & 0.401 \\
\hline 2 & 0.032 & 0.026 & 0.038 & 0.037 & 0.045 & 0.043 & 0.038 & 0.046 & 0.033 & 0.041 & 0.032 & 0.033 \\
\hline 3 & 0.277 & 0.183 & 0.329 & 0.278 & 0.382 & 0.379 & 0.342 & 0.399 & 0.194 & 0.229 & 0.178 & 0.175 \\
\hline 4 & 1.000 & 1.000 & 1.000 & 1.000 & 1.000 & 1.000 & 1.000 & 1.000 & 1.000 & 1.000 & 1.000 & 1.000 \\
\hline 5 & 0.108 & 0.098 & 0.102 & 0.103 & 0.038 & 0.040 & 0.049 & 0.064 & 0.151 & 0.146 & 0.289 & 0.290 \\
\hline 6 & 0.426 & 0.351 & 0.403 & 0.481 & 0.705 & 0.703 & 0.739 & 0.705 & 0.466 & 0.463 & 0.222 & 0.202 \\
\hline 7 & 0.036 & 0.033 & 0.035 & 0.032 & 0.022 & 0.022 & 0.027 & 0.031 & 0.043 & 0.039 & 0.051 & 0.048 \\
\hline 8 & 0.500 & 0.438 & 0.483 & 0.450 & 0.319 & 0.316 & 0.328 & 0.395 & 0.350 & 0.355 & 0.383 & 0.383 \\
\hline 9 & 0.553 & 0.477 & 0.685 & 0.681 & 0.571 & 0.571 & 0.525 & 0.577 & 0.400 & 0.341 & 0.405 & 0.412 \\
\hline 10 & 0.088 & 0.079 & 0.112 & 0.115 & 0.113 & 0.114 & 0.088 & 0.089 & 0.076 & 0.059 & 0.074 & 0.076 \\
\hline 11 & 1.206 & 1.016 & 1.376 & 1.216 & 1.451 & 1.408 & 1.104 & 1.023 & 0.505 & 0.520 & 0.688 & 0.678 \\
\hline
\end{tabular}


Table 4: Calculated similarity of communion peak

\begin{tabular}{|c|c|c|c|c|c|c|c|c|c|c|c|c|c|}
\hline Code & $\overline{S 1}$ & $\overline{\text { S2 }}$ & $\overline{S 3}$ & S4 & S5 & 56 & S7 & S8 & S9 & S10 & S11 & S12 & Reference \\
\hline S1 & 1.000 & 0.754 & 0.985 & 0.756 & 0.885 & 0.752 & 0.752 & 0.634 & 0.903 & 0.721 & 0.773 & 0.878 & 0.854 \\
\hline S2 & 0.754 & 1.000 & 0.755 & 0.980 & 0.751 & 0.821 & 0.617 & 0.790 & 0.771 & 0.695 & 0.959 & 0.960 & 0.875 \\
\hline S3 & 0.985 & 0.755 & 1.000 & 0.759 & 0.677 & 0.749 & 0.748 & 0.853 & 0.899 & 0.646 & 0.871 & 0.874 & 0.863 \\
\hline S4 & 0.756 & 0.980 & 0.759 & 1.000 & 0.688 & 0.855 & 0.952 & 0.756 & 0.776 & 0.743 & 0.940 & 0.939 & 0.885 \\
\hline S5 & 0.885 & 0.751 & 0.677 & 0.688 & 1.000 & 0.966 & 0.969 & 0.615 & 0.749 & 0.890 & 0.792 & 0.884 & 0.915 \\
\hline S6 & 0.752 & 0.821 & 0.749 & 0.855 & 0.966 & 1.000 & 0.997 & 0.804 & 0.675 & 0.832 & 0.936 & 0.829 & 0.918 \\
\hline S7 & 0.752 & 0.617 & 0.748 & 0.952 & 0.969 & 0.997 & 1.000 & 0.898 & 0.978 & 0.830 & 0.934 & 0.728 & 0.926 \\
\hline S8 & 0.634 & 0.790 & 0.853 & 0.756 & 0.615 & 0.804 & 0.898 & 1.000 & 0.853 & 0.939 & 0.867 & 0.961 & 0.930 \\
\hline s9 & 0.903 & 0.771 & 0.899 & 0.776 & 0.749 & 0.675 & 0.978 & 0.853 & 1.000 & 0.870 & 0.930 & 0.727 & 0.820 \\
\hline S10 & 0.721 & 0.695 & 0.646 & 0.743 & 0.890 & 0.832 & 0.830 & 0.939 & 0.870 & 1.000 & 0.716 & 0.710 & 0.831 \\
\hline S11 & 0.773 & 0.959 & 0.871 & 0.940 & 0.792 & 0.936 & 0.934 & 0.867 & 0.930 & 0.716 & 1.000 & 0.998 & 0.815 \\
\hline $\mathrm{S} 12$ & 0.878 & 0.960 & 0.874 & 0.939 & 0.884 & 0.829 & 0.728 & 0.961 & 0.727 & 0.710 & 0.998 & 1.000 & 0.848 \\
\hline reference & 0.854 & 0.875 & 0.863 & 0.885 & 0.915 & 0.918 & 0.926 & 0.930 & 0.820 & 0.831 & 0.815 & 0.848 & 1.000 \\
\hline
\end{tabular}

0.80. The result demonstrated that each of the twelves G. rhizoma samples had a great similarity compared with the reference chromatogram. Therefore, the quality of twelves G. rhizoma samples from three different provinces (Sichuan, Guizhou and Shanxi) in China were similar. Nevertheless, we discovered some tiny differences between the samples from different province that the similarity of Guizhou, Shanxi, Sichuan samples were of $0.854-0.885$, $0.915-0.930$ and $0.820-0.848$, respectively. In contrast, the similarities of samples from different province didn't have a certain regularity.

The eleven characteristic peaks in chromatograms were the main components of $G$. rhizoma. These three provinces are main produced places of $G$. rhizoma in China, so the quality of three area can represent the average quality in China. As a result, for quality control of G. rhizoma in China, 0.800 was set as a fit value. That is to say, the samples of G. rhizoma have reliable quality when the similarity was above 0.800 .

\section{Hierarchical cluster}

The RPA of 11 characteristic peaks of twelves samples were analyzed by the SPSS software. The distances among the twelve samples were calculated via the SPSS software, with the between-groups linkage as the cluster method and the squared Euclidean distance as the measure interval. The result of experiment suggested that the twelves samples could be divided into three clusters at the distance of 7.5: the samples of S1-S4 can be classified as cluster 1 ; the samples of S5-S8 can be formed as cluster 2; the others samples can be grouped into cluster 3 . The relationships among the $G$. rhizoma samples collected from three different provinces in China is shown in the dendrogram which constructed according to HCA (Figure 4).

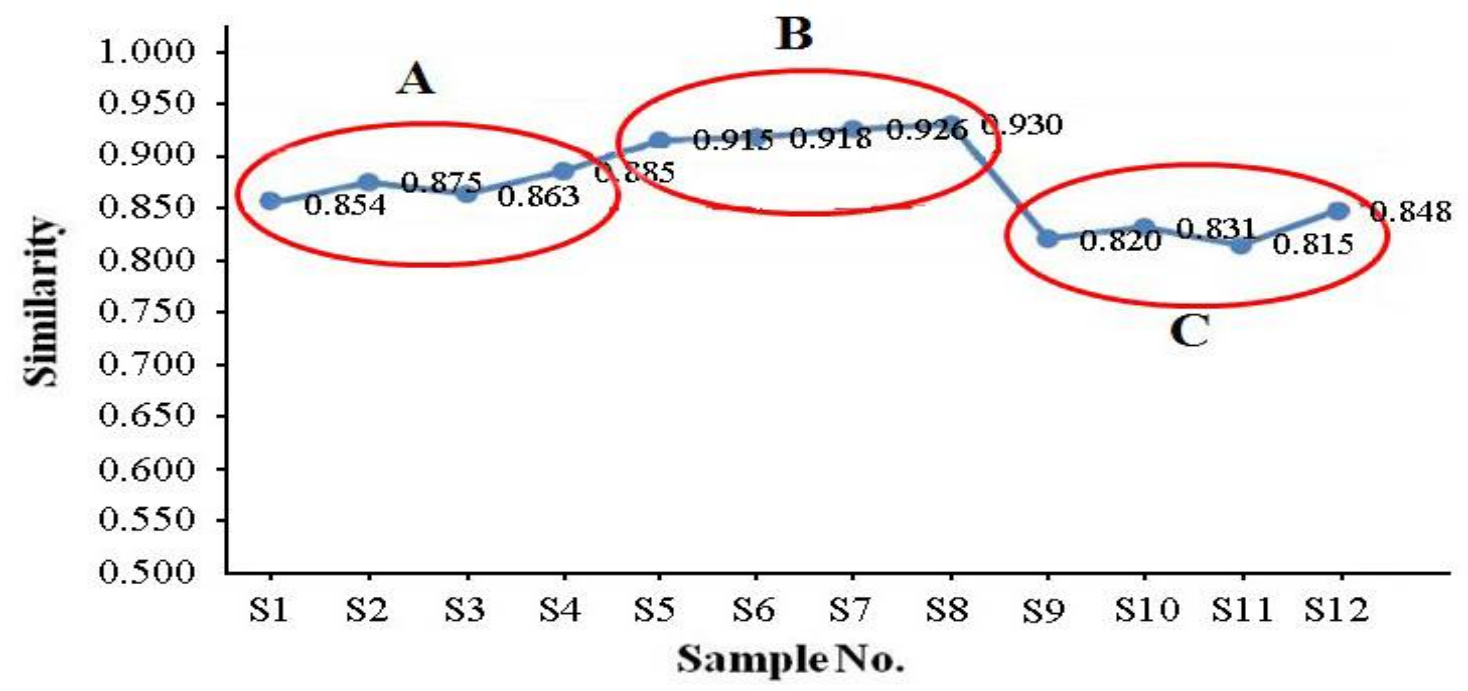

Figure 3: Line chart of similarity analysis 


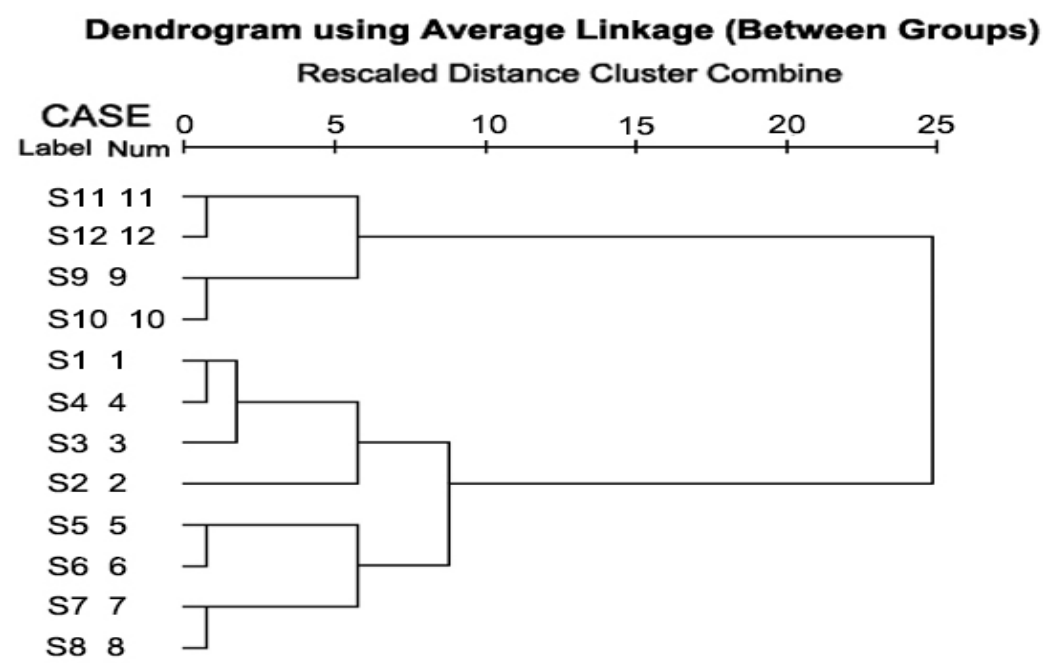

Figure 4: Results of hierarchical cluster analysis of twelve samples

From Figure 4, the twelve samples were categorized into three groups based on HCA. The samples from cluster 1 (Guizhou) and cluster 2 (Shanxi) were of more similarity than the samples from cluster 3 (Sichuan). The classification results by HCA indicated that the sample from the same area had more similar chemical fingerprints and internal quality than others. The classification based on HCA was consistent with the similarity analysis results of G. rhizoma (Figures 3 and 4), and the results can mutually authenticate. The results can provide a new method for origin discrimination and quality evaluation of $\mathrm{G}$. rhizoma.

\section{DISCUSSION}

G. rhizoma, a precious herbal medicine of TCM, has been used for a long time in China to treat hypertension, reduce dizziness, improve circulation, and relieve headaches $[15,16]$. As the increasing consumption of G. rhizoma, its safety and quality are being increasingly concerned $[17,18]$. Therefore, it is urgent to develop a high efficacy method for the origin discrimination and quality control of $G$. rhizoma from different origins.

HPLC fingerprint is emerging as a viable technique for qualitative and quantitative analysis of natural products, and the applications in the analysis of TCM has been also reported in the literature [19-21]. Similarity analysis and HCA are popular methods in applied statistical work and data analysis, and they have a good ability to summarize multivariate variation. With their help, the relationship between the fingerprints and the origins of $G$. rhizoma was elucidated in the present study. Thus, an HPLC fingerprint method has been established for the evaluation and origin discrimination of G. rhizoma collected from three different areas in China.

The chromatographic data for the twelve samples were processed by similarity analysis and HCA. From the results of the similarity analysis, we can find that the G. rhizoma samples have relatively constant quality, and the HPLC fingerprint method could be used in the quality control. Interestingly, the samples from three major origins of $G$. rhizoma have some differences. In addition, the results of HCA indicated the presence of a correlation between the major origin area of $G$. rhizoma samples and the types and quantities of chemical constituents.

\section{CONCLUSION}

HPLC fingerprint and origin discrimination method for $G$. rhizoma from three areas in China have been developed and established. The method and established model are efficient and accurate, and can be applied in the quality control and major origin area discrimination of $G$. rhizoma.

\section{DECLARATIONS}

\section{Acknowledgement}

This work was supported by Fund of the National "Twelfth Five-Year Plan" Science \& Technical Projects (no. 2012BAI29B11) and State Administration of Traditional Chinese Medicine of the People's Republic of China (no. 2010070123-6).

\section{Conflict of Interest}

No conflict of interest associated with this work. 


\section{Contribution of Authors}

The authors declare that this work was done by the authors named in this article and all liabilities pertaining to claims relating to the content of this article will be borne by them.

\section{REFERENCES}

1. Editorial Committee of Chinese Pharmacopoeia. Chinese pharmacopoeia. Vol. 1. China Chemical Industry Press: Beijing; 2015; $p 58$.

2. Ha JH, Lee DU, Lee JT, Kim JS, Yong CS, Kim JA, Ha JS, Huh K. 4-Hydroxybenzaldehyde from Gastrodia elata B1. is active in the antioxidation and GABAergic neuromodulation of the rat brain. $J$ Ethnopharmacol 2000; 73(1-2): 329-333.

3. Jung JW, Yoon BH, Oh HR, Ahn JH, Kim SY, Park SY, Ryu JH. Anxiolytic-like effects of Gastrodia elata and its phenolic constituents in mice. Biol Pharm Bull 2006; 29(2): 261-265.

4. Li HB1, Chen F. Preparative isolation and purification of gastrodin from the Chinese medicinal plant Gastrodia elata by high-speed counter-current chromatography. J Chromatogr A 2004; 1052(1-2): 229-232.

5. Cao $Y$, Zhang $X$, Fang $Y, Y e J$. Determination of the active ingredients in Gastrodia rhizoma by capillary electrophoresis with electrochemical detection. Analyst. 2001; 126(9): 1524-1528.

6. Tao J, Luo ZY, Msangi Cl, Shu XS, Wen L, Liu SP, Zhou $C Q$, Liu RX, Hu WX. Relationships among genetic makeup, active ingredient content, and place of origin of the medicinal plant Gastrodia tuber. Biochem Genet. 2009; 47(1-2): 8-18.

7. Xu S, Yang L, Tian R, Wang Z, Liu Z, Xie P, Feng Q. Species differentiation and quality assessment of Radix Paeoniae Rubra (Chi-shao) by means of highperformance liquid chromatographic fingerprint. J Chromatogr A 2009; 1216(11): 2163-2168.

8. Xie $P$, Chen $S$, Liang YZ, Wang $X$, Tian $R$, Upton $R$. Chromatographic fingerprint analysis--a rational approach for quality assessment of traditional Chinese herbal medicine. J Chromatogr A 2006; 1112(1-2): 171180.

9. Ma T, Huang $C$, Meng $X$, Zhang $Q$, Zhang $L, L v X$, Jin $Y$, Xie J, Li J. Fingerprint analysis of Hawk-tea by highperformance liquid chromatography. Food Chem 2011, 129: $551-556$.

10. Ye J, Zhang X, Dai W, Yan S, Huang H, Liang X, Li Y, Zhang $W$. Chemical fingerprinting of Liuwei Dihuang Pill and simultaneous determination of its major bioactive constituents by HPLC coupled with multiple detections of DAD, ELSD and ESI-MS. I Pharm Biomed Anal 2009; 49(3): 638-45.

11. Xu L, Han X, Qi Y, Xu Y, Yin L, Peng J, Liu K, Sun C. Multiple compounds determination and fingerprint analysis of Lidanpaishi tablet and keli by highperformance liquid chromatography. Anal Chim Acta 2009; 633(1): 136-148.

12. Gong F, Liang YZ, Xie PS, Chau FT. Information theory applied to chromatographic fingerprint of herbal medicine for quality control. J Chromatogr A. 2003; 1002(1-2): 25-40.

13. Ch PC. Chinese pharmacopoeia, Vol. 1. Beijing: Chinese medical science and technology press; 2010. Appendix 130p.

14. Cao $Y$, Zhang $X$, Fang $Y, Y e ~ J$. Determination of the active ingredients in Gastrodia rhizoma by capillary electrophoresis with electrochemical detection. Analyst 2001; 126(9): 1524-1528.

15. Tang C, Wang L, Liu $X$, Cheng $M$, Xiao $H$. Pharmacokinetic study of Gastrodia elata in rats. Anal Bioanal Chem 2015; 407(29): 8903-8910.

16. Wang N, Wu TX, Zhang $Y, X u X B$, Tan S, Fu HW. Experimental analysis on the main contents of Rhizoma gastrodiae extract and inter-transformation throughout the fermentation process of Grifola frondosa. Arch Pharm Res 2013; 36(3): 314-321.

17. Zhu $Y$, Zheng Q, Sun Z, Chen Z, Zhao $Y$, Wang Z, Yang $H$, Li J, Li Y, Xiao X. Fingerprint-efficacy study of Radix Aconiti Lateralis Preparata (Fuzi) in quality control of Chinese herbal medicine. J Therm Anal Calorim 2014; 118: 1763-1772.

18. Chen L, Song F, Liu Z, Zheng Z, Xing J, Liu S. Study of the ESI and APCI interfaces for the UPLC-MS/MS analysis of pesticides in traditional Chinese herbal medicine. Anal Bioanal Chem. 2014; 406(5): 1481-1491.

19. Zhu H, Wang C, Qi Y, Song F, Liu Z, Liu S. Fingerprint analysis of Radix Aconiti using ultra-performance liquid chromatography-electrospray ionization/ tandem mass spectrometry (UPLC-ESIMS n) combined with stoichiometry. Talanta. 2013; 103: 56-65.

20. Dong L, Cheng B, Luo Y, Zhang N, Duan H, Jiang $M$, Wang $Y$, Bai G, Luo G. Identification of nuclear factor-KB inhibitors and $\beta 2$ adrenergic receptor agonists in Chinese medicinal preparation Fuzilizhong pills using UPLC with quadrupole time-of-flight MS. Phytochem Anal 2014; 25(2): 113-121.

21. Fang $L X$, Xiong AZ, Wang $R$, Ji S, Yang L, Wang ZT. A strategy for screening and identifying mycotoxins in herbal medicine using ultra-performance liquid chromatography with tandem quadrupole time-of-flight mass spectrometry. J Sep Sci. 2013; 36(18): 31153122. 